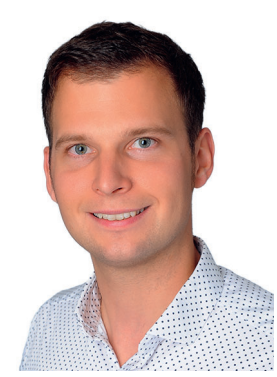

\title{
Merkmale und Risikofaktoren eines diagnostizierten und nicht diagnostizierten Trockenen Auges, identifiziert mithilfe einer Smartphone-App
}

Sebastian Siebelmann

Zentrum für Augenheilkunde, Uniklinik Köln, Köln, Deutschland

Zusammenfassung zu Inamata T, Iwagami M, Nakamura M, et al.: Characteristics and risk factors associated with diagnosed and undiagnosed symptomatic dry eye using a smartphone application. JAMA Ophthalmol. 2019;DOI:10.1001/jamaophthalmol.2019.4815

\section{Zusammenfassung}

Ziel der Studie war es, mithilfe der Smartphone-App DryEyeRhythm Charakteristika und Risikofaktoren bei diagnostiziertem und nicht diagnostiziertem Trockenen Auge zu identifizieren. Dazu wurden die Daten der App-User gesammelt und analysiert (vorliegende Informationen schlossen ein: Demografie, medizinische Vorgeschichte, Informationen zur Lebenweise der Nutzer, subjektive und krankheitsspezifische Symptome erhoben mithilfe des Ocular Surface Disease Index und der Zung Self-Rating Depression Scale). Insgesamt wurden die Daten von 4454 Nutzern (899 mit diagnostiziertem und 2395 mit nicht diagnostiziertem Trockenen Auge) anaysiert und folgende Risikofaktoren für ein symptomatisches Trockenes Auge identifiziert: junges
Alter, weibliches Geschlecht, Heuschnupfen, Depression, psychische Erkrankungen außer Depression oder Schizophrenie, momentanes Tragen von Kontaktlinsen, lange Bildschirmarbeit und Rauchen. Risikofaktoren für ein nicht diagnostiziertes symptomatisches Trockenes Auge umfassten: junges Alter, männliches Geschlecht, kein Vorliegen einer Kollagenose, psychische Erkrankungen außer Depression oder Schizophrenie, ophthalmologische chirurgische Eingriffe außer LASIK oder Kataraktoperation und Kontaktlinsennutzung (momentan oder in der Vergangenheit). Risikofaktoren für ein nicht diagnostiziertes, nicht symptomatisches Trockenes Auge konnten nicht identifiziert werden.

c 2019 S. Karger GmbH, Freiburg 


\section{Transfer in die Praxis}

\section{Hintergrund}

Die Inzidenz des Trockenen Auges steigt in den letzten Jahrzehnten deutlich an. Gründe dafür sind hauptsächlich Umweltfaktoren, wie Bildschirm-Arbeitsplätze oder Klimaanlagen, können aber auch autoimmune Erkrankungen wie das Sjögren-Syndrom sein. Generell sind die Symptome eines Trockenen Auges eher unspezifisch und erstrecken sich vom typischen «müden Auge» über Brennen, Tränen, Verschwommensehen bis hin zum Blepharospasmus. Aufgrund dieser Vielzahl an möglichen Beschwerden bleibt bei einer großen Anzahl der Patienten das Trockene Auge meist undiagnostiziert. Die hier vorgestellte Studie nutzt eine SmartphoneApp (DryEyeRythm), um anhand einer großen Datenbank Patienten mit einem Trockenen Auge zu identifizieren, indem per App ein OSDI-Fragebogen (Ocular Surface Disease Index) ausgefüllt wurde. Zudem sollten Gemeinsamkeiten unter den Patienten, die an einem Trockenen Auge leiden, aufgedeckt werden. Darüber hinaus untersucht die Studie Merkmale von Patienten mit einem Trockenen Auge, bei denen dieses bereits vorher durch einen Augenarzt diagnostiziert worden war, mit denen, bei denen, deren Erstdiagnose durch die App erfolgte [1].

\section{Dry Eye Rhythm}

DryEyeRhythm ist eine App, die von der Jutendo Universität Tokio entwickelt wurde und auf dem iPhone und dem iPad läuft Mit ihrer Hilfe soll es laut Herstellerangaben möglich sein, innerhalb von 5 Minuten und einem Fragebogen ein Trockenes Auge ohne echten Patientenkontakt zu diagnostizieren. Neben aus gewählten Informationen zum Trockenen Auge bietet die App noch eine Erfassung und Analyse von Schrittzahlen, der Monitorzeit, der Auswertung des Schlafs, von Stress sowie die Erfassung von Augenschmerzen. Die Auswertung der so erhobenen Daten an einer großen Anzahl an Patienten könnte in Zukunft interessante Erkenntnisse zum Zusammenspiel unterschiedlicher Faktoren zur Entstehung des Trockenen Auges liefern. Insbesondere ist hier die Auswertung mittels künstlicher Intelligenz hervorzuheben, die es vermag, große Datenpools und unterschiedliche Arten erfasster Daten zu kombinieren. Auch vor dem Hintergrund, dass inzwischen Apps therapeutisch in das Leben von Patienten eingreifen und in Zukunft mehr und mehr von den Krankenkassen erstattet werden könnten, ist die hier vorgestellte Studie besonders interessant.

\section{Ergebnisse der Studie}

Mithilfe der in der Studie gewonnen Daten war es generell möglich Patienten mit einem symptomatischen Trockenen Auge zu identifizieren und Risikofaktoren für ein symptomatisches Trockenes Auge darzustellen. Diese umfassten vor allem junges Alter, das Tragen von Kontaktlinsen, Heuschnupfen bzw. die okuläre Allergie, Depressionen sowie weitere psychische Erkrankungen, Rauchen und eine verlängerte Bildschirmarbeitsplatzzeit. Risikofaktoren da- für an einem symptomatischen Trockenen Auge zu leiden, welches zuvor nicht diagnostiziert wurde, waren dahingegen psychische Erkrankungen außer der Depression und der Schizophrenie und vorangegangene Augenoperationen außer der LASIK und der Katarakt-Operation. Eine weitere interessante Erkenntnis ist, dass ein männliches Geschlecht offensichtlich ein Risikomerkmal für ein nicht diagnostiziertes Trockenes Auge ist und als solches eine Art Barriere für die korrekte Diagnose eines solchen darstellt. Als Kritik an der Studie ist festzustellen, dass vermutlich das junge Alter als Risikofaktor für ein symptomatisches Trockenes Auge an der Rekrutierung der Studienteilnehmer mithilfe des Mediums Smartphone-App liegt und somit als Stichprobenverzerrung anzusehen ist. Eine weitere Stichprobenverzerrung stellt das Studiendesign mittels einer App dar, die nur über das iOS-Betriebssystem von Apple ausführbar war, da sich diese Anwendergruppe teils von den Nutzern des Android-Betriebssystems unterscheidet. Darüber hinaus war es anhand des Studiendesigns nicht möglich ein undiagostiziertes, nicht symptomatisches Trockenes Auge zu diagnostizieren, da die Ergebnisse der Studie nicht mit echten klinischen Daten korreliert wurden, sondern sich ausschließlich auf den OSDI stützen. Dennoch gibt es auch am OSDI durchaus Kritik, da dieser sich hauptsächlich auf unspezifische Symptome stützt [2].

\section{Fazit für die Praxis}

Die Identifikation von Patienten mit einem Trockenen Auge könnte auch als Ferndiagnose mittels Smartphone-App und ohne direkten Patientenkontakt möglich sein. Dabei können zudem Risikofaktoren identifiziert und anhand von Big Data ausgewertet werden und gegebenenfalls kann sogar im Sinne einer Therapie auf einzelne Pathogenesefaktoren Einfluss genommen werden. In Zukunft könnten Smartphone-Apps sowohl bei der Diagnose als auch bei der Therapie von ausgewählten Erkrankungen wie dem Trockenen Auge zunehmend an Bedeutung gewinnen.

\section{Disclosure Statement}

Der Autor hat keine finanziellen Interessen.

\section{Literatur}

1 Inomat T, Iwagami M, Nakamura M, et al.: Characteristics and risk factors associated with diagnosed and undiagnosed symptomatic dry eye using a smartphone application. JAMA Ophthalmology. 2020;138:58-68.

2 Amparo F, Schaumberg DA, Dana R: Comparison of two questionnaires for dry eye symptom assessment: the ocular surface disease index and the symptom assessment in dry eye. Ophthalmology. 2015;122;1498-1503.

Kontaktadresse: PD Dr. Sebastian Siebelmann, Zentrum für Augenheilkunde, Uniklinik Köln, Kerpener Straße 62, 50937 Köln, Deutschland, sebastian.siebelmann@uk-koeln.de 